\title{
Facial Reconstruction and Cosmetology - A Review
}

\author{
Monisha B. $\mathbf{M}^{1}$, Abirami Arthanari ${ }^{2}$, Pushpalatha ${ }^{3}$, Priyadharshini $\mathbf{R}^{4}$, Palati Sinduja ${ }^{4}$ \\ ${ }^{1}$ Assistant Professor, Skin and Std. Department, Vinayaka Missions Kirupananda Variyar Medical College and \\ Hospitals, Salem, Tamilnadu, ${ }^{2}$ Lecturer, Dept.of Forensic Odontology, ${ }^{3}$ Tutor, Dept.of Implantology, Saveetha \\ Dental College and Hospital, Saveetha Institute of Medical and Technical Sciences (SIMATS) Chennai - 77, \\ Tamilnadu, ${ }^{4}$ Senior lecturer, Department of Pathology, Saveetha Dental College, Saveetha Institute of Medical and \\ Technical Sciences (SIMATS), Chennai 77, TamilNadu
}

\begin{abstract}
Facial reconstruction can be used to identify unknown human skeletal remains when other techniques be unsuccessful. Through this review, we try to assessment the different methods of facial reconstruction stated in literature. There are several techniques of doing facial reconstruction, which vary from two dimensional drawings to three-dimensional clay models. With the advancement in 3D technology, a rapid, efficient and cost-effective computerized 3D reconstruction method has been developed which has brought down the grade of mistake before come across. Recognition allows the involved administration activities to make a list of assumed victims. This list can then be pointed down and a positive identification may be given by the more conventional method of forensic field. Facial reconstruction allows visual identification by the individual's family and associates to become easy and more definite. And here cosmetic reconstruction also plays a best role in investigating the criminals and legal part.
\end{abstract}

Key Words: Forensic Facial Reconstruction, Cosmetic Reconstruction, 3D Techniques

\section{Introduction}

Facial reconstruction is presently utilized in 2 principal contexts: rhetorical science and archaeology. within the rhetorical context it plays a very important role in identification of the dead wherever postmortem deterioration has created this problematical. In archaeology, it's wont to produce three-dimensional visual pictures of individuals from the past, from

\footnotetext{
Corresponding Author

Dr. Abirami Arthanari,

B.D.S, M.Sc, PhD (On-Going)

Lecturer, Dept.of Forensic Odontology,

Saveetha Dental College and Hospital,

Saveetha Institute of Medical and Technical Sciences

(SIMATS) Chennai - 77, Tamilnadu

Mobile: 7904652975
}

skeletal remains, mummified bodies, or bodies preserved. Facial reconstruction aims at sick the facial look of a private from the only real information of the underlying bone. The facial reconstruction drawback arises in varied application fields like forensics, social science, archaeology or history. In rhetorical science, facial reconstruction comes within the method of identification of deceased folks. once all the standard ways of identification have failing and therefore the skeletal remains are the only real knowledge offered for resulting in an identification, facial reconstruction may be thought of as Associate in Nursing enhancing tool for "recognition", manufacturing a brief list of candidates from that the individual could also be known by different supported ways of identification. the standard facial reconstruction ways are supported manual procedures, manufacturing 2 nd portraits or $3 \mathrm{D}$ sculptures. These ways essentially encompass 3 common steps: (1) equip 
(a duplicate of) the raw bone with a thin set of anatomical landmarks; (2) apply a mean soft tissue thickness to every bone landmark so as to estimate a corresponding landmark on the face; (3) draw up or sculpt a face fitting the calculable landmarks. Most practitioners add a face muscle model so as to complement the anatomical accuracy of the reconstruction, resulting in the supposed Manchester technique delineate in ${ }^{[1]}$.

The reconstruction of enormous surgical defects when the surgical excision of skin tumours typically needs the utilization of skin flaps. For optimum results, it's vital to require into consideration the idea of facial cosmetic units. ${ }^{[2]}$ shown in figure one

\section{Methods of 3D reconstruction}

1. Measuring Method/ Tissue Depth Method:

This was developed by Krogman in 1946 . Through this technique, soft tissue depth knowledge is taken into account. This technique was usually used for reconstruction by enforcement agencies. Fine measurements were obtained by the utilization of needles, X-rays or ultrasound. As facial muscles are recorded in a very correct anatomical manner, this technique needs extremely trained personnel, thus this system isn't most well-liked currently a day. ${ }^{[1]}$

\section{Anatomical Russian Method:}

This technique was developed by Gerasimov in 1971. Here soft tissue depth knowledge wasn't thought of however facial muscles were utilized in anatomical position. during this technique, reconstruction was done by shaping muscles, glands and animal tissue onto the bone layer by layer. this system isn't usually utilized in recently. This technique is far slower than the Russian technique and a larger degree of anatomical data is needed. Reconstruction of fossilised skulls are achieved by this technique. ${ }^{[2]}$

3. Combination Manchester Method/ British Method:

This technique was developed by Neave in 1977 and is that the most accepted technique for facial reconstruction these days. during this technique, each soft tissue thickness and facial muscles are taken into thought. Once the so Associate in Nursing the articulator is articulated and therefore the bone is mounted on an adjustable exchange the Frankfort Horizontal plane, tissue pegs or markers are then additional on the bone, either by inserting them directly on the bone or by inserting them on antecedental trained holes on the solid at ninety degree employing a $3 \mathrm{~mm}$ drilling bit. every peg length represents the mean tissue depth at the anatomical purpose. The tissue depth is decided by the age, gender, build etc. of the individual. The muscles of chew and face expression are made out of the modelling material and positioned on the premise of their origin and insertion on the bone. the form and size of assorted muscles are determined on the premise of the underlying laborious tissues. Plaster or prosthetic plastic eye balls of $25 \mathrm{~mm}$ diameter are placed into the orbits. The prosthetic eye balls are positioned within the orbit in such the simplest way in order that a tangent taken from the middle bodily cavity margin to the middle infraorbital margins touches the iris. The inner corner is placed $2 \mathrm{~mm}$ lateral to the lacrimal crest and therefore the outer corner is placed $4 \mathrm{~mm}$ medial to the malar tubercle. once the malar tubercles are absent, the outer corner is placed $10 \mathrm{~mm}$ below the road of frontozygomatic suture and $5-7 \mathrm{~mm}$ from the orbital margin. the utmost dimension of the nose is decided by the bony nasal aperture at its widest purpose as three-fifths of the general dimension of the soft nose. The profile of the nose and therefore the form and therefore the size of the alae are determined by the nasal aperture. The jaw canine and 1st bicuspid are placed close to the corners of mouth and dimension of the mouth corresponds to 6 anterior teeth. The thickness of lips is decided by higher and lower anterior teeth. The length of the ear is foretold by the length of the nose and therefore the auditory canal is positioned by victimisation external meatus because the point of reference. Muscles of the face are typically modelled on the bone that is to be reconstructed in clay one by one, then a layer of clay is additional over the muscle system to represent the skin, hypodermic fats and strips of clay 
are then rolled, formed and additional over the muscle/ fat structure to make the finished face by maintaining the length of the pegs as a guide to the ultimate tissue guides over the face. ${ }^{[2]}$

\section{Computerised 3D rhetorical Facial} Reconstruction:

With the advancement in 3D technology, a fast, economical and cost-efficient pc assisted rhetorical facial reconstruction technique was developed. during this technique, the operator used $3 \mathrm{D}$ computerised model's victimisation manual clay model techniques.
Some computerised systems used 3D animation package to model the face onto the bone whereas different system used virtual sculpture system with soma to sense feedback. Some to sense feedback system has the power to feel the surface of the bone throughout analysis and conjointly give vital skeletal details for facial reconstruction like muscle attachment strength, position of eye, position of malar tubercle etc. this method although needs each social science and pc modelling skills. It decreases practitioner's sound judgement and ability. This technique conjointly creates multiple pictures of constant face quickly and expeditiously. ${ }^{[3]}$
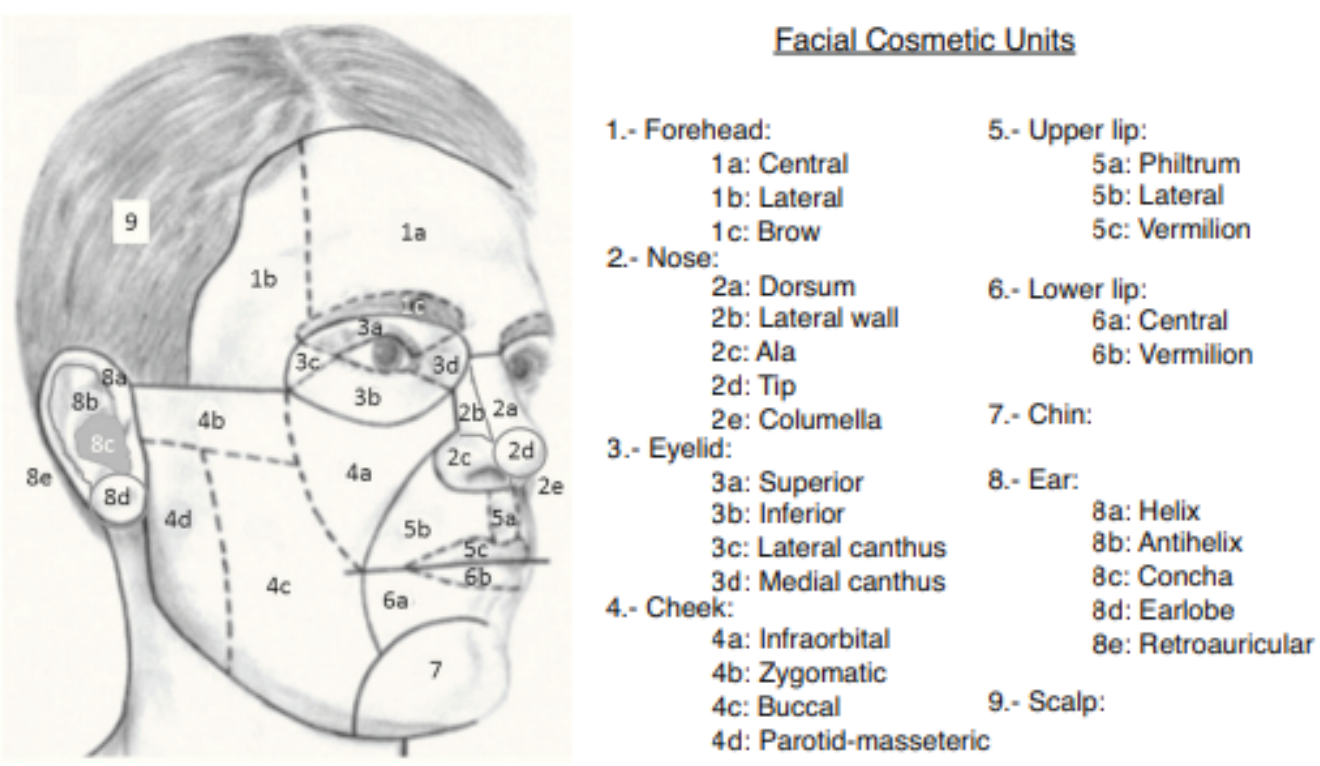

Fig 1: Cosmetic Unit and Its Sub - Units

There are 3 facial reconstruction flaps conjointly present:

1) First alternative of reconstruction flaps

2) Second alternative of reconstruction flaps

3) Third alternative of reconstruction flaps

All the reconstruction techniques chosen by every rhetorical skilled and a medical specialist for every facial reconstruction and cosmetic reconstruction are listed so as of preference within the further Material, in conjunction with the ultimate score obtained ffor every for every\} reconstruction technique in each cosmetic fractional monetary unit. ${ }^{[4,5,6]}$

\section{Conclusion}

Scientific testing is that the solely approach forward and far of it's required. the shortage of systematic scientific tests within the past has enabled several dishonest notions to become established. several of those notions seem to own arisen and been sustained as a results of professional person biases - this is often 
clearly evident even within the name usually want to describe the strategy of building faces from skulls, for "facial reconstruction" implies everything the strategy. though facial approximation ways are helpful for rhetorical investigation and cosmetic procedures, the general public ought to watch out for the promoting and political ploys utilized inside the profession.

Ethical Clearance- Since it is a Review article, we did not take ethical

Source of Funding- Self

\section{Conflict of Interest - Nil}

\section{References}

1. Fernandes CM, Pereira FD, da Silva JV, Serra Mda C. Is characterizing the digital forensic facial reconstruction with hair necessary? A familiar asssessors' analysis. Forensic Sci Int. 2013;
229:164. e1-e5.

2. Stephan $\mathrm{C}$ and Henneberg M. Bullding faces from dry skulls: Are they recognized above chance rates, Journal of Forensic Sciences 2001; 46: 432440.

3. Wilkinson C. Computerized forensic facial reconstruction: a review of current systems. Forensic Sci Med Pathol. 2005; 1:173-177.

4. Naftel AJ, Mao Z. Acquiring dense 3D facial models using structured light assisted stereo correspondence TRS-2002-004-ajn. UMIST Department of Communication 2002.

5. Russo F, Linares M, Iglesias ME, Martínez-Amo JL, Cabo F, Tercedor J, et al. Técnicas reconstructivas de elección por unidades estéticas faciales. Actas Dermosifiliogr. 2017;108:729---737.

6. Thomas CL, Lam A, Lam J, Paver R, Storey L, FernandezPeñas $\mathrm{P}$, et al. Factors affecting choice of repair in mohs micrographic surgery for nonmelanoma skin cancer of the head. Australas J Dermatol 2017;58:189-93. 